\title{
ACTIVITÉ COCGIDIOSTATIQUE D'UN COMPLEXE SULFATE DE FRAMYCETINE MÉNADIONE BISULFITIQUE
}

\author{
PAR
}

\author{
J. AYCARDI \\ Station de Recherches avicoles C.N.R.Z., Jouy-en-Josas (S. et O.).
}

\section{SOMMAIRE}

Le sulfate de framycétine, en addition continue à la ration, possède une activité préventive intéressante dans la coccidiose caecale du poulet. La dose de roo $\mathrm{g}$ p. tonne s'est montrée la plus efficace. L'association de cet antibiotique avec la ménadione bisulfitique à I $g$ p. tonne d'aliment constitue un complexe coccidiostatique très satisfaisant. Il permet une inhibition énergique du développement des parasites, une amélioration sensible de la croissance en milieu contaminé et un indice de consommation favorable. L'immunité consécutive à l'infestation parasitaire n'est pas entravée. Aucun signe d'intolérance ne se manifeste chez les animaux traités.

L'activité anticoccidienne curative de la framycétine - antibiotique extrait de cultures de Streptomyces Sp. Decaris - mise en évidence par LuCAS et a1., I956, a été confirmée par différents auteurs (LABatu' et al., I958), (PERdRIx, I958), (Aycardi, I959). L'administration prolongée de cette substance n'ayant pas d'effet fâcheux pour l'animal (inocuité) et ne présentant pas de danger pour le consommateur (absence de stockage tissulaire chez le poulet), il était souhaitable d'étudier l'activité coccidiostatique éventuelle de la framycétine introduite de façon continue dans les aliments pour volailles. D'autre part, le choc hémorragique consécutif à la coccidiose caecale du poulet, bien étudié par NatT et HERrick, I955, est largement compensé par l'addition, à la ration, de vitamine $\mathrm{K}$ ('TUgweIL, et a1., I957). La ménadione bisulfitique se montre à cet égard nettement plus efficace que la ménadione (Goldhaft et Wernicoff, 1954), (Scotr, I954), (Frost et Spruth, I955), (O'rro et al., r958). La diminution sensible de la mortalité par 
coccidiose dépend uniquement de l'activité antihémorragique et non d'une éventuelle activité anticoccidienne.

Nous rapportons ici les résultats d'une série d'expériences mettant en évidence l'activité coccidiostatique préventive du sulfate de framycétine et l'intérêt de son association avec la ménadione bisulfitique.

Les essais ont porté sur 2 I lots de poussins représentant au total 960 animaux élevés en batteries standard du commerce modifiées selon les besoins expérimentaux (isolement des 1ots, récupération des excrétats, contrôle des quantités d'eau et d'aliments consommée, etc.). Chaque lot expérimental comportait en début d'expérience, 40 ou 50 poussins d'un jour (des deux sexes) d'un croisement chair du commerce Sussex $\times$ Rhode $\times$ Wyandotte. A chaque lot fut opposé un lot témoin de même effectif et même provenance. I a répartition des individus dans les différents lots fut faite selon le poids à ro jours et de telle façon que la dispersion et la moyenne des poids soient identiques pour tous les lots à comparer.

Pendant les 2 premiers jours d'âge, tous les animaux ont été alimentés au maïs. Puis les aliments poussins supplémentés ont été distribués de façon continue, à partir du $3^{\mathrm{e}}$ jour et jusqu'à la fin de chaque expérience. L'aliment poussin utilisé est un aliment standard, sans incorporation d'aucun supplément (antibiotiques, condiments, antioxydants) autres que les suppléments à expérimenter.

Nature et doses des divers suppléments expérimentés :

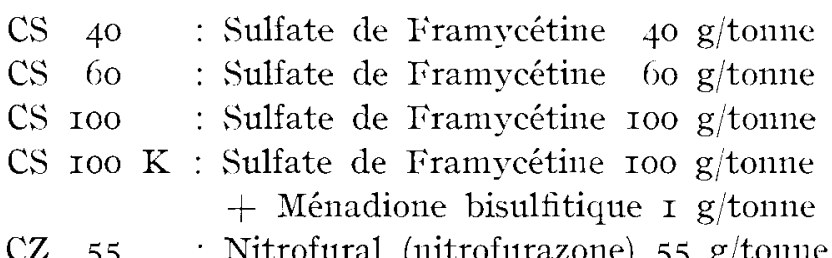

CZ 55 : Nitrofural. (nitrofurazone) $55 \mathrm{~g} /$ tonne

Ce dernier dosage à titre de comparaison (coccidiostat couramment utilisé dans la pratique).

Pour chaque expérience la même méthode a été utilisée. Elle s'appuie sur les observations et mesures suivantes:

- Mortalité par coccidiose au cours des différents périodes d'épreuve.

- Numération des ookystes de coccidies dans les excréments totaux de 24 heures - pour chaque lot expérimental - du $6^{\mathrm{e}}$ au $2 \mathrm{I}^{\mathrm{e}}$ jour qui suivent l'inoculation d'épreuve.

- Autopsie et examen parasitologique de tous les animaux morts en cours d'expérience; éventuellement examen histologique.

- Croissance, appréciée par les gains de poids vif (pesées individuelles).

- Taux de conversion alimentaire. 
Les épreuves furent de deux ordres :

Io Épreuve d'efficacité $=$ Ire inoculation.

Au $\mathrm{I}^{\mathrm{e}}$ jour, chaque poussin reçoit individuellement dans le jabot, 50000 ookystes sporulés d'Eimeria tenella (souche entretenue par passages successifs sur poussins). A chaque lot traité est opposé un lot témoin inoculé, non traité C'T et un lot témoin non inoculé et non traité S'T.

\section{$2^{\mathrm{o}}$ Épreuve d'immunité $=2^{\mathrm{e}}$ inoculation.}

$\mathrm{Au} 43^{\mathrm{e}}$ jour, une inoculation semblable à la précédente mais comportant Ioo ooo ookystes, est pratiquée. Elle a pour but d'apprécier l'immunité acquise, vis-à-vis de la coccidiose caecale, à la suite de la

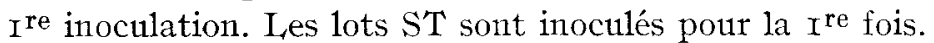

L'analyse des courbes d'élimination des ookystes montre que l'efficacité anticoccidienne (fig. I) croît à mesure que la dose de sulfate de framycétine augmente. En particulier 1'association CS roo K réalise une excellente inhibition du développement des parasites et une atténuation rapide de l'infestation dans le temps. Ce dernier point est important à considérer car la seconde période d'élimination $\left(\mathrm{I}_{5}^{\mathrm{e}}\right.$ aul $2 \mathrm{I}^{\mathrm{e}}$ jour après l'inoculation) rend compte de la prolongation du parasitisme dans le temps et par conséquent de la persistance des lésions. Les courbes relatives aux lots $\mathrm{CS}$ roo et $\mathrm{CS}$ Ioo $\mathrm{K}$ se superposent étroitement. Nous n'avons représenté que celle de CS Ioo $\mathrm{K}$ afin de ne pas surcharger le graphique. Comparativement, le nitrofural a une activité moindre et surtout moins régulière. L'élimination dure plus longtemps.

On peut également exprimer l'activité coccidiostatique sous la forme du taux d'inhibition relatif $\mathrm{T}=\frac{e_{t}-e_{i}}{e_{t}} \times$ Ioo par rapport au témoin inoculé non traité correspondant, avec:

$e_{t}=$ nombre d'ookystes éliminés par le lot témoin inoculé, $e_{i}=$ nombre d'ookystes éliminés par le lot traité.

Les taux relatifs moyens ainsi calculés (moyeine de $I_{5}$ taux relatifs successifs portant sur 20 numérations chacun) sont rapportés dans le tableau ci-contre. Le taux moyen d'inhibition dans les lots CS roo K est de 87,5 p. roo, ce qui représente un contrôle énergique du parasitisme : les animaux traités éliminent en moyenne 8 fois moins d'ookystes que les témoins C'T. Le nitrofural est moins actif $(\overline{\mathrm{T}}=68,3)$.

I.'établissement et le maintien de l'immunité qui suit la première inoculation sont mis en évidence par les courbes d'élimination obtenues lors de la seconde épreuve (fig. 2) ainsi que par les taux d'inhibition calculés comme précédemment (cf. tableau) mais cette fois par rapport à ST. Tous les lots traités ont une immunité du même ordre que celle des témoins C'T (immunité naturelle). 'Toutefois les lots CS roo K éliminent 


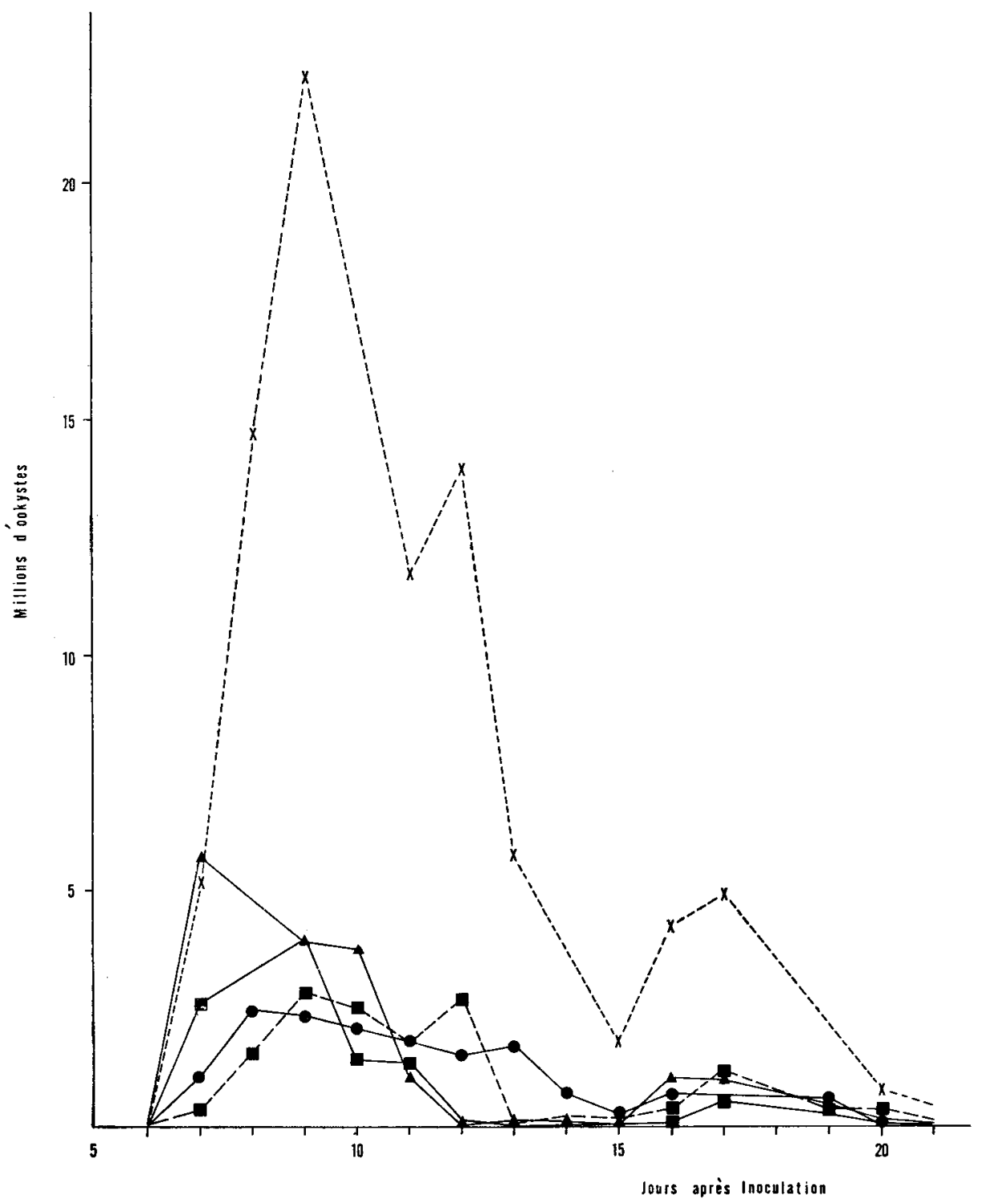

FIG. r. - Élimination quotidienne d'Ookystes par animal, dans les fécès. $\mathrm{I}^{\mathrm{re}}$ inoculation (Efficacité)

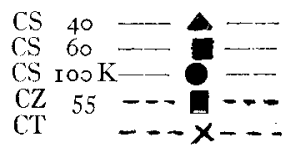


(9, I960) COCCIDIOSTA'TS : FRAMYCÉ'TINE-MÉNADIONE

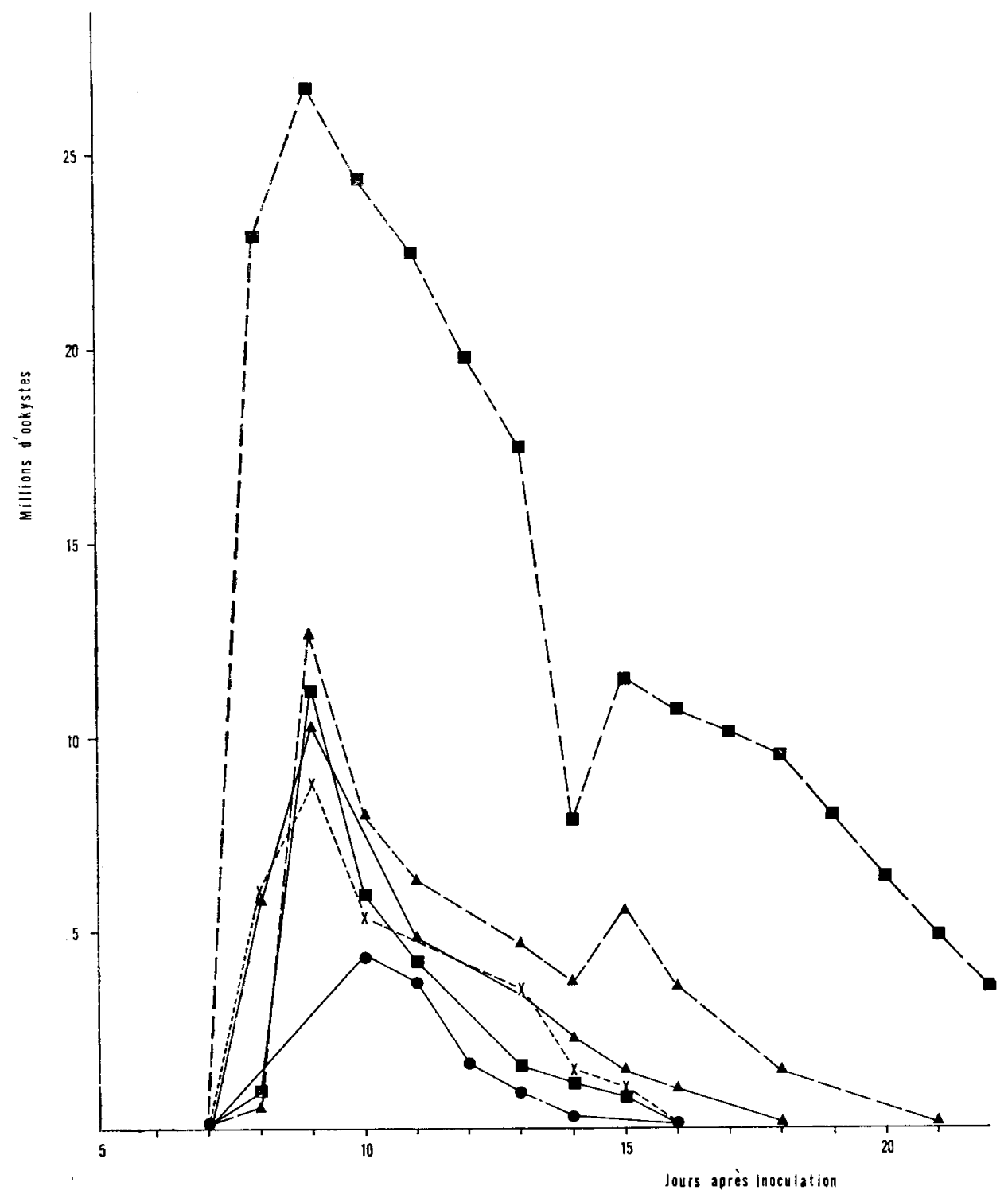

FIG 2. - Élimination quotidienne d'Ookystes par animal, dans les fécès. $2^{\mathrm{e}}$ inoculation (Immunité).

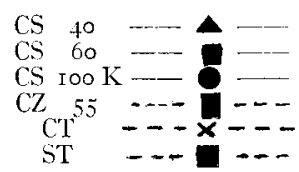


nettement moins d'ookystes $(\overline{\mathrm{T}}=9 \mathrm{I}, 9)$, et surtout moins longtemps. Le nitrofural ne permet qu'une immunité médiocre $(\overline{\mathrm{T}}=73,7)$.

Les données relatives à la mortalité, à la croissance et au taux de conversion alimentaire sont rapportées dans le tableau ci-contre. Elles confirment la supériorité des lots CS Ioo K. Les gains de poids au cours des deux épreuves (efficacité et immunité) y sont exprimés en p. Ioo des témoins C'T et S'T correspondants. De ce point de vue, les lots CS Ioo K sont significativement différents des lots $\mathrm{C} T$ (gain total de + I3,9 p. Ioo) et supérieurs à tous les autres lots. L'absence de toxicité chronique est ici, parallèlement à 1'activité coccidiostatique, un facteur important dans le maintien d'une croissance correcte et dans l'obtention d'indices de consommation favorables.

\section{TABLEAU II}

Activité coccidiostatique du sulfate de framycétine seul ou en association avec la ménadione bisulfitique dans la coccidiose caecale du poulet.

\begin{tabular}{|c|c|c|c|c|c|c|c|c|}
\hline & Traitements & $\operatorname{CS}_{40}$ & $\operatorname{CS} 60$ & CS 100 & $\begin{array}{c}\mathrm{CS} \\
\mathrm{I} 00 \mathrm{~K}\end{array}$ & $C Z 55$ & $\mathrm{CT}$ & $\mathrm{ST}$ \\
\hline $\begin{array}{l}\text { Épreuve } \\
\text { d'efficacité }\end{array}$ & $\begin{array}{l}\text { Taux d'inhibition relatif } \\
\text { par rapport à CT } \ldots \ldots \\
\text { Mortalité p. Ioo } \ldots \ldots \\
\text { Gain de poids } \\
\text { en p. Ioo CT } \ldots \ldots \ldots \\
\text { en p. Ioo ST } \ldots \ldots \ldots\end{array}$ & $\begin{array}{c}76,7 \\
2 \\
104,3 \\
92,7\end{array}$ & $\begin{array}{c}83,3 \\
2 \\
\\
\text { I I } 1,9 \\
99,4\end{array}$ & $\begin{array}{c}84, \mathrm{I} \\
\mathrm{I} \\
\text { I } 18,2 \\
106,5\end{array}$ & $\begin{array}{c}87,5 \\
0 \\
\text { I } 16,9 \\
105,4\end{array}$ & $\begin{array}{c}68,3 \\
0 \\
108,1 \\
96,0\end{array}$ & $\begin{array}{l}- \\
- \\
-\end{array}$ & $\begin{array}{l}- \\
- \\
-\end{array}$ \\
\hline $\begin{array}{l}\text { Épreuve } \\
\text { d'immunité }\end{array}$ & $\begin{array}{l}\text { Taux d'inhibition relatif } \\
\text { par rapport à ST } \ldots \ldots \\
\text { Mortalité p. Ioo } \ldots \ldots \\
\text { Gain de poids } \\
\text { en p. Ioo CT . . . . . } \\
\text { en p. Ioo ST } \ldots \ldots \ldots\end{array}$ & $\begin{array}{c}84,5 \\
2 \\
107,0 \\
108,8\end{array}$ & $\begin{array}{c}90,1 \\
0 \\
\\
1 C 9,3 \\
\text { I I I, I }\end{array}$ & $\begin{array}{c}92, \mathrm{I} \\
0 \\
100,8 \\
102,4\end{array}$ & $\begin{array}{c}91,9 \\
0 \\
\text { I09,9 } \\
\text { I I 1 }, 6\end{array}$ & $\begin{array}{c}73,7 \\
6 \\
\\
99,4 \\
\text { IOI, I }\end{array}$ & $\begin{array}{c}87,6 \\
0 \\
-\end{array}$ & $\begin{array}{l}10 \\
--\end{array}$ \\
\hline Total & 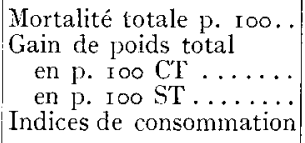 & $\begin{array}{c}4 \\
\text { I } 05,6 \\
99,8 \\
2,78\end{array}$ & $\begin{array}{c}2 \\
\text { I I } 0,6 \\
\text { I } 04,6 \\
2.6 \text { I }\end{array}$ & $\begin{array}{c}\mathrm{r} \\
\text { I } 10,5 \\
\text { I04,8 } \\
2,+0\end{array}$ & $\begin{array}{l}\circ \\
\text { II } 3,9 \\
\text { IO8,0 } \\
\quad 2,42\end{array}$ & $\begin{array}{r}6 \\
\\
104,0 \\
98,3 \\
2,51\end{array}$ & $\begin{array}{l}4 \\
- \\
\frac{-}{2,58}\end{array}$ & $\begin{array}{l}10 \\
- \\
2,34\end{array}$ \\
\hline
\end{tabular}

L'association sulfate de framycétine-ménadione bisulfitique présente done un réel intérêt comme adjuvant alimentaire dans les aliments pour volailles et satisfait aux conditions habituellement requises pour un bon coccidiostatique : efficacité, respect de l'immunité, absence de toxicité, action favorable sur la croissance et sur l'indice de consommation en milieu contaminé de coccidiose. 


\section{REMERCIEMENTS}

Nous tenons à remercier les Usines Chimiques des Laboratoires Français et l'institut Professionnel de Contrôle et de Recherches Scientifiques des Industries de 1'Alimentation Animale pour la contribution matérielle qu'ils ont apportée à la réalisation de ce travail.

\section{SUMMARY}

An experiment dealing with 960 birds has shown the coccidiostatic activity of framycetine sulfate when added continually to the feed of broilers. The dose of roo $\mathrm{g} /$ ton has been shown to be the most active. The association of this antibiotic with menadione sodium bisulfite in doses of $\mathrm{Ig} /$ ton energetically controls parasitism, improves growth ( + I3,9 p. Ioo of the controls) and gives satisfactory feed efficiency. There was no sign of toxicity throughout the various treatments.

\section{REFERENCES BIBLIOGRAPHIQUES}

AyCARDr J., I959. Étude de différentes substances pouvant être utilisées contre la coccidiose et la pullorose du poussin en traitement préventif et curatif. Industr. Alimentat. anim., 93, $27-32$.

Frost D. V. et SPruth H. C., I955. Control of haemorrhagic condition in chickens with Menadione sodium bisulfite. Poult. Sci., 34, 56-64.

GoIDHAFT T. M. et WERNICOFF N., 1954. A report on a haemorthagic condition occuring in poultry in the United States. Xe Congr. Mondial Avic. Edimbourg, Section C. Paper $n^{0} 84,278-282$.

Iabatut R., Camguilhem $H$. et Cantan R., I958. Essais de traitement de l'amidostomose et de la coccidiose chez les Oies. Rev. Med. Vét., 109, 730-737.

Lucas A., Toucas I., Laroche M., I956. Un antibiotique doué de propriétés anticoccidiennes : la Framycétine. Rec. Méd. Vét. Alfort, 132, Q25-928.

NATT M. P. et HERrick C. A., I955. The Effect of caecal coccidiosis on the blood cells of the domestic fowl. Poult. Sci., 34, I I00-I.Io6.

Otтo G. F., Jeske H. A., Frost I). V. et Perdue H. S., I958. Menadione sodium bisulfite complex (Klotogen F) in caecal coccidiosis. Poult. Sci., 37, 20I-205.

PERDRIX J., I958. Hémorragies consécutives au traitement de la coccidiose aviaire par la framycétine. Rec. Med. Tét. Alfort., 134, 8Ig-820.

ScotT H. M., I954. Kansas State formula feed. Conf.

Tugweil R. L., Stephens J. F. et Harms R. H., I957. The relationship of vitamin $\mathrm{K}$ to mortality from caecal coccidiosis. Poult. Sci., 36, I245I 247 . 